\title{
Imaging of early stage breast cancer with circularly polarized light
}

Dremin, Viktor, Anin, Dmytro, Sieryi, Oleksii, Borovkova, Mariia, Näpänkangas, Juha, et al.

Viktor Dremin, Dmytro Anin, Oleksii Sieryi, Mariia Borovkova, Juha Näpänkangas, Igor Meglinski, Alexander Bykov, "Imaging of early stage breast cancer with circularly polarized light," Proc. SPIE 11363, Tissue Optics and Photonics, 1136304 (7 April 2020); doi: 10.1117/12.2554166

SPIE. Event: SPIE Photonics Europe, 2020, Online Only, France 


\title{
Imaging of early stage breast cancer with circularly polarized light
}

\author{
Viktor Dremin ${ }^{\mathrm{a}, \mathrm{b}}$, Dmytro Anin ${ }^{\mathrm{a}}$, Oleksii Sieryi ${ }^{\mathrm{a}}$, Mariia Borovkova $^{\mathrm{a}}$, \\ Juha Näpänkangas ${ }^{c}$, Igor Meglinski ${ }^{\mathrm{a}, \mathrm{d}, \mathrm{e}}$, and Alexander Bykov ${ }^{\mathrm{a}}$ \\ ${ }^{a}$ Optoelectronics and Measurement Techniques Unit, University of Oulu, Oulu, Finland \\ ${ }^{\mathrm{b}}$ Research \& Development Center of Biomedical Photonics, Orel State University, Orel, Russia \\ ${ }^{c}$ Oulu University Hospital, University of Oulu, Oulu, Finland \\ ${ }^{\mathrm{d}}$ School of Engineering \& Applied Science, Aston University, Birmingham, UK

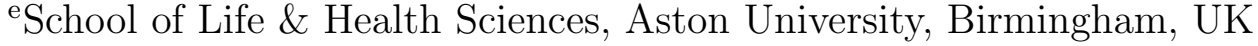

\begin{abstract}
In this study, we utilize the properties of polarized light for the analysis of paraffin-embedded breast cancer samples. We perform the measurements of the full Stokes vector of back-reflected radiation and calculate the degree of polarization as a diagnostic criterion for the separation of healthy and cancer sample sections. We show that circularly polarized light scattered within the breast sample is sensitive to the presence of cancer cells. The degree of the polarization of the reflected light was found to be the most sensitive parameter for the reliable differentiation of tissue.
\end{abstract}

Keywords: breast cancer, Stokes polarimetry, polarimetric imaging, degree of polarization

\section{INTRODUCTION}

Breast carcinoma is the most widespread cancer among women with more than 400000 cases diagnosed each year across EU countries. ${ }^{1}$ For successful treatment, the early detection of cancer is extremely important. Millions of women are surviving and thriving with breast cancer when found and treated early. Currently, the only way and gold standard to set definite diagnostics of cancer is histology. However, despite the best laboratory practices, the rate of conclusive diagnosis by histological analysis for a range of cancers is only $65-75 \% .^{2}$ Typically, the biopsy is taken at the time of surgery operation with a high demand for immediate qualitative pathological review. The development of an efficient diagnostic modality may revolutionize the current practice of cancer diagnosis and treatment. The ability to recognize the suspect tissue in a short time without the interruption of the operation is able to increase the effectiveness of the surgery of the cancerous tumors.

Many imaging studies use the polarized light as a supplementary tool e.g. to reduce the specular reflection from the surface of the measured object. ${ }^{3}$ However, recent research ${ }^{4-12}$ demonstrates the potential of bulk polarimetry as a standalone modality for the biotissue diagnosis. In this study, we suggest using the properties of polarized light for the analysis of formalin-fixed paraffin-embedded (FFPE) breast cancer samples. We propose measurements of the Stokes vector and the calculation of the degree of polarization (DOP) as a diagnostic criterion for the separation of healthy and cancer sample sections. The standard routine procedure for the histological analysis uses a number of sample preparation steps, which include fixation of the sample in formalin, ethanol, etc.; dehydration and embedding into the paraffin wax or celloidin; sectioning with a microtome; mounting on glass slides, staining by hematoxylin, eosin, etc.; and finally microscopy investigation. ${ }^{13}$ Thus, the conventional histological analysis is rather laborious, time-consuming and strongly relies on the skills and qualification of the pathologist performing the examination. However, the proposed approach allows us to avoid several steps in the conventional histology procedure and perform the tissue sample analysis right after the embedding step.

Send correspondence to Viktor Dremin

E-mail: viktor.dremin@oulu.fi,

Telephone: +358414706340

Tissue Optics and Photonics, edited by Valery V. Tuchin, Walter C. P. M. Blondel,

Zeev Zalevsky, Proc. of SPIE Vol. 11363, 1136304 - (c) 2020 SPIE

CCC code: $0277-786 \mathrm{X} / 20 / \$ 21 \cdot$ doi: $10.1117 / 12.2554166$

Proc. of SPIE Vol. $113631136304-1$ 

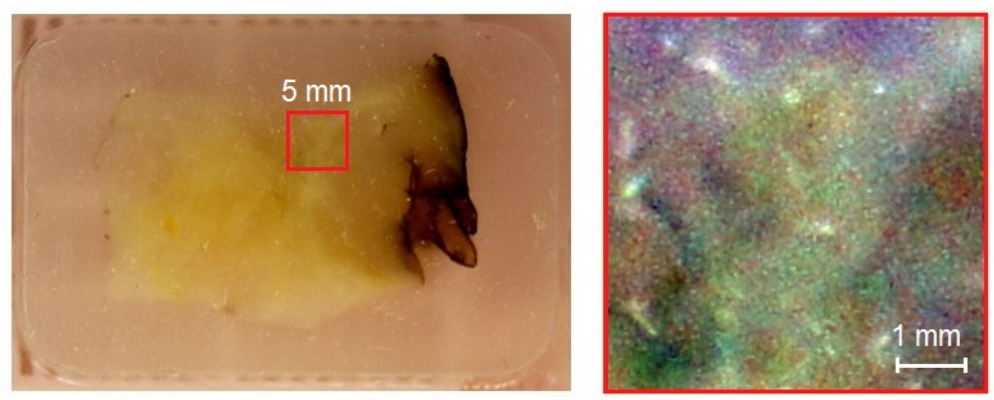

Figure 1. Overview of FFPE breast cancer samples (left side) with ROI showing the enlarged area scanned with microscope (right side).

As you can see from Fig. 1, standard microscopic examination of the paraffin-embedded tissue sample does not provide any useful diagnostic information due to the low contrast between tissue constituents in the unstained sample. In the same time, the involvement of the polarization properties sensitive to the changes in the microstructure of the sample can solve this problem as well as provide quantitative information about the analyzed sample. In addition, our approach will reduce both the number of sample processing steps and the time needed for the analysis.

\section{MATERIAL AND METHODS}

Due to the high sensitivity of polarized light to the variations of scattering in the analyzed sample, the spatially resolved polarized light spectroscopy has a great potential to be used for screening of malformations in the biological tissues. ${ }^{14}$ We have developed an experimental polarization-based imaging system for scanning biological samples. In our setup, circularly polarized light was used due to its unique ability to preserve the polarization properties during larger number of scattering events than the linearly polarized light. ${ }^{15,16}$ The measurement system contains both illumination and detection channels (see Fig. 2).

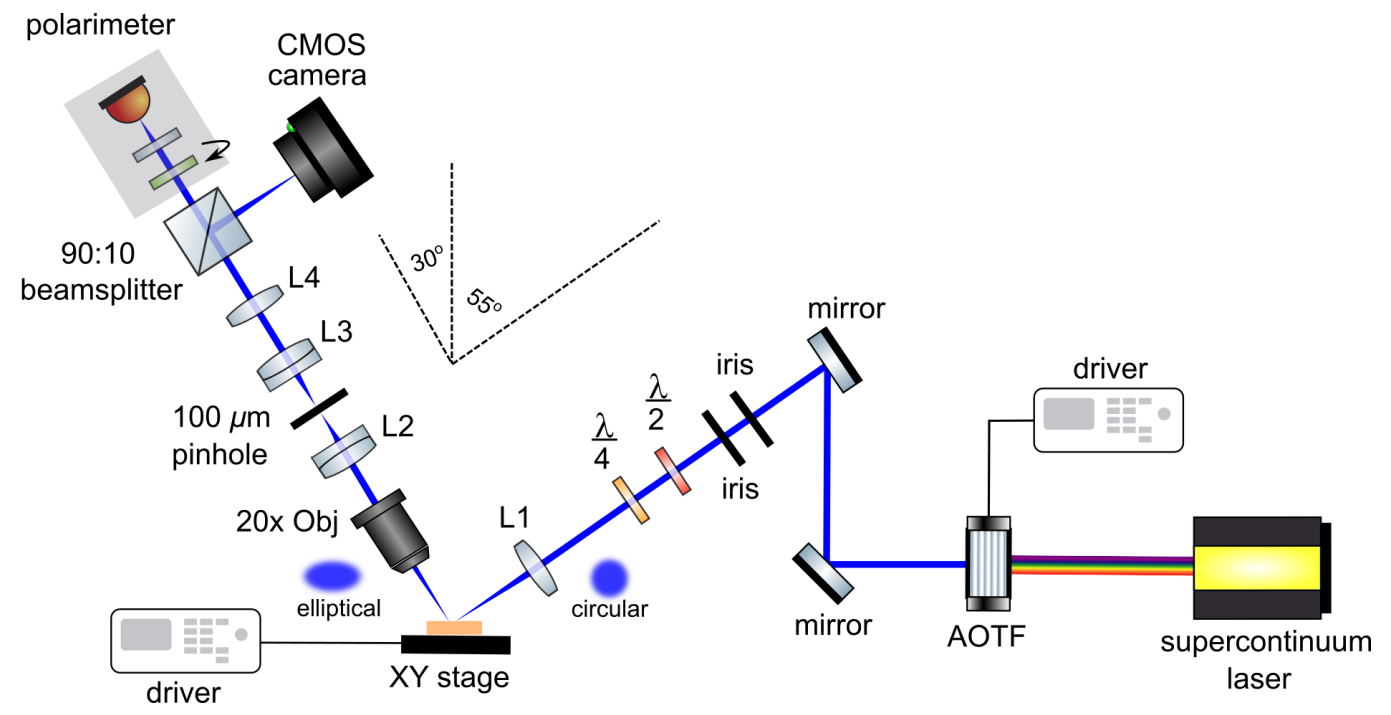

Figure 2. Scheme of the experimental setup containing the illumination arm (right side) and measurement arm (left side).

The radiation of supercontinuum fiber laser (Leukos Ltd., France) was used in the illumination channel. The specific probing wavelength was selected with the high-speed acousto-optic tunable filter (Leukos Ltd., France) from the range of $400-650 \mathrm{~nm}$. The linearly polarized light from the output of the filter was then altered by a 
quarter-wave plate into the state of right-hand circular polarization and focused onto the sample at $55^{\circ}$ angle. The state of polarization of the light diffusely reflected from the sample was collected at $30^{\circ}$ angle at a variable distance away from the point of incidence with the $20 \times$ objective lens and analyzed by the Stokes polarimeter system (Thorlabs Ltd., USA). Spatial scanning of the samples was performed with the XY translation stage providing the resolution of $5 \mu \mathrm{m}$.

The multiple measurements have been performed on a FFPE blocks of early stage breast cancer samples (ductal carcinoma in situ, DCIS) and compared to the measurements from developed cancer samples (invasive ductal carcinoma, IDC). Breast cancer samples and their histology were provided by the Northern Finland Biobank Borealis (Oulu, Finland).

\section{RESULTS AND DISCUSSION}

We show that circularly polarized light scattered within the breast sample is sensitive to the presence of cancer cells and provides the information close to that obtained from the standard histological examination (see Fig. 3). However, no staining and no slicing is required in our case.
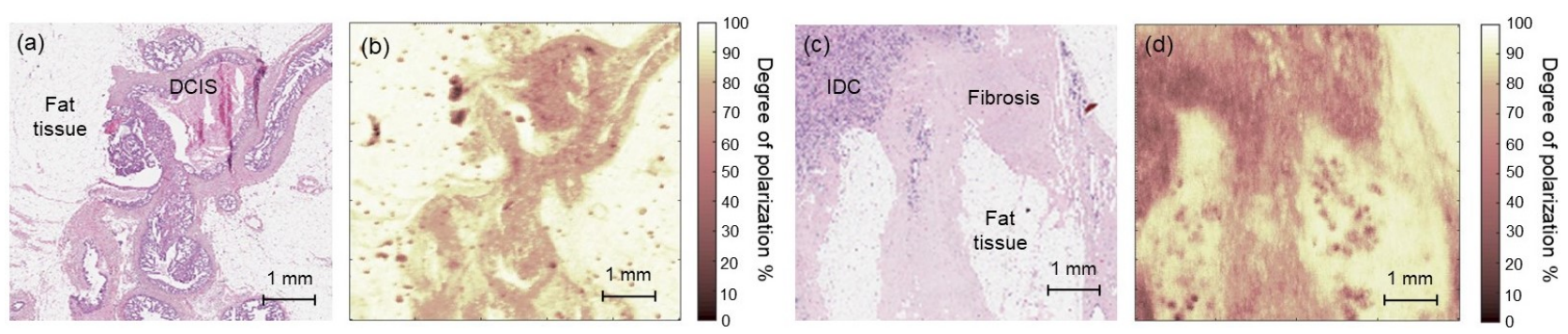

Figure 3. Histological image of DCIS (a) and 2D spatial distributions of the degree of polarization of the reflected light measured at $450 \mathrm{~nm}$ (b). Histological image of IDC and benign fibrosis (c) and 2D spatial distributions of the degree of polarization (d).

The highest contrast between cancerous and normal regions was observed for the probing wavelength of 450 $\mathrm{nm}$. The DOP of the reflected light was found to be the most sensitive parameter indicating the malignant changes in the considered samples. DOP shows how much of light is polarized within the whole light that is detected. The average DOP values for intact adipose tissue were $95 \%$, for DCIS - 70-80\%, the presence of IDC reduced the DOP value to the level of $60-70 \%$. The proposed methodology demonstrates a great potential for developing a new diagnostic protocol for routine clinical practice.

And finally, a statistically significant differences confirmed by Kruskal-Wallis test with the post-hoc analysis $(p<0.01)$ exists between four types of tissue in the DOP parameter. The observed differences may be related to an increase in the average size of the nuclei in a more developed type of cancer. This leads to higher anisotropy and an increase in successive forward scattering events, which reduces the DOP.

\section{CONCLUSION}

A circular polarization imaging system for obtaining histological images was developed. We have shown than circularly polarised light scattered within breast samples is sensitive to the presence of cancer cells and the highest perceptual contrast between cancerous and normal regions was observed for the probing wavelength of $450 \mathrm{~nm}$. The DOP of the reflected light was found to be the most sensitive parameter indicating the malignant changes in the considered samples. The proposed methodology demonstrates a great potential for developing a new diagnostic protocol for routine clinical practice. 


\section{ACKNOWLEDGMENTS}

Authors acknowledge the support of the Academy of Finland (grants: 314369 and 325097), the ATTRACT project funded by the EU under Grant Agreement 777222. This study was also supported by the Russian Science Foundation under projects No. 19-79-00082 (experimental data collection). Authors acknowledge Northern Finland Biobank Borealis, Oulu, Finland (www.ppshp.fi/biopankki) for providing the breast cancer samples.

\section{REFERENCES}

[1] [Health at a Glance: Europe 2018: State of Health in the EU Cycle], OECD Publishing, Paris (2018).

[2] Bray, F., Ferlay, J., Soerjomataram, I., Siegel, R. L., Torre, L. A., and Jemal, A., "Global cancer statistics 2018: Globocan estimates of incidence and mortality worldwide for 36 cancers in 185 countries," $C A: A$ Cancer Journal for Clinicians 68(6), 394-424 (2018).

[3] Zherebtsov, E., Dremin, V., Popov, A., Doronin, A., Kurakina, D., Kirillin, M., Meglinski, I., and Bykov, A., "Hyperspectral imaging of human skin aided by artificial neural networks," Biomed. Opt. Express 10(7), 3545-3559 (2019).

[4] Novikova, T., Meglinski, I., Ramella-Roman, J., and Tuchin, V., "Polarized light for biomedical applications," J. Biomed. Opt. 21(7), 071001 (2016).

[5] Ushenko, V., Sdobnov, A., Syvokorovskaya, A., Dubolazov, A., Vanchulyak, O., Ushenko, A., Ushenko, Y., Gorsky, M., Sidor, M., Bykov, A., and Meglinski, I., "3D Mueller-matrix diffusive tomography of polycrystalline blood films for cancer diagnosis," Photonics. 5, 54 (2018).

[6] Ushenko, A., Sdobnov, A., Dubolazov, A., Gritsuk, M., Ushenko, Y., Bykov, A., and Meglinski, I., "Stokescorrelometry analysis of biological tissues with polycrystalline structure," IEEE J. Sel. Top. Quantum Electron. 25(1), 7101612 (2019).

[7] Borovkova, M., Ushenko, V. A., Dubolazov, A. V., Vanchulyak, O. Y., Ushenko, O. G., Bykov, A. V., and Meglinski, I., "Mueller-matrix-based polarization imaging and quantitative assessment of optically anisotropic polycrystalline networks," PLOS One 14(5), e0214494 (2019).

[8] Borovkova, M., Peyvasteh, M., Ushenko, Y. O., Dubolazov, O. V., Ushenko, V. O., Bykov, A. V., Novikova, T. P., and Meglinski, I., "Complementary analysis of Muller-matrix images of optically anisotropic highly scattering biological tissues," J. Eur. Opt. Soc.-Rapid. 14, 20 (2018).

[9] Ushenko, V. A., Sdobnov, A. Y., Mishalov, W. D., Dubolazov, A. V., Olar, O. V., Bachinskyi, V. T., Ushenko, A. G., Ushenko, Y. A., Wanchuliak, O. Y., and Meglinski, I., "Biomedical applications of jonesmatrix tomography to polycrystalline films of biological fluids," J. Innov. Opt. Heal. Sci. 12(6), 1950017 (2019).

[10] Ushenko, V. A., Dubolazov, A. V., Pidkamin, L. Y., Sakchnovsky, M. Y., Bodnar, A. B., Ushenko, Y. A., Ushenko, A. G., Bykov, A., and Meglinski, I., "Mapping of polycrystalline films of biological fluids utilizing the Jones-matrix formalism," Laser Phys. 28(2), 025602 (2018).

[11] Das, N. K., Dey, R., Chakraborty, S., Panigrahi, P. K., Meglinski, I., and Ghosh, N., "Quantitative assessment of submicron scale anisotropy in tissue multifractality by scattering Mueller matrix in the framework of born approximation," Opt. Commun. 413, 172-178 (2018).

[12] Das, N. K., Dey, R., Chakraborty, S., Panigrahi, P. K., Meglinski, I., and Ghosh, N., "Submicron scale tissue multifractal anisotropy in polarized laser light scattering," Laser Phys. Lett. 15(3), 035601 (2018).

[13] Nozdrin, V., Belousova, T., Piavchenko, G., and Volkov, Y., [A concise guide to histology], Retinoids, Moscow (2019).

[14] Kunnen, B., Macdonald, C., Doronin, A., Jacques, S., Eccles, M., and Meglinski, I., "Application of circularly polarized light for non-invasive diagnosis of cancerous tissues and turbid tissue-like scattering media," J. Biophotonics. 8, 317-323 (2015).

[15] Xu, M. and Alfano, R., "Circular polarization memory of light," Phys. Rev. E 72(6), 065601 (2005).

[16] MacKintosh, F., Zhu, J., Pine, D., and Weitz, D., "Polarization memory of multiply scattered light," Phys. Rev. B 40(13), 9342 (1989). 